\title{
Research on the Enumerating Equation of Rectilinear Embedding- Counting Rooted Spherical Near Quadrangulations
}

\author{
Liyan Pan ${ }^{1, *}$ and Yanpei Liu $^{2}$ \\ ${ }^{1}$ College of Business, Shanghai Jianqiao University, Shanghai, 201306, China \\ ${ }^{2}$ College of Science, Beijing Jiaotong University, Beijing, 100044, China \\ ${ }^{*}$ Corresponding author
}

\begin{abstract}
This paper provides quartic functional equations satisfied by the enumerating functions of rooted planar near-quadrangulations with the size, the valency of the root-face and the number of non-rooted vertices. Rooted two edgeconnected planar near-quadrangulations are also counted. The quartic and the cubic functional equations are proposed for the first time, furthermore, explicit formulae for such two types of maps with above parameters are derived respectively after employing Lagrangian inversion. For two particular cases, the numbers of rooted planar trees and outerplanar quadrangulations are deduced directly. The studying results are helpful for rectilinear embedding in VLSI(Very Large Scale Integration), for the Gaussian crossing problem in graph theory, for the knot problem in topology, and for the enumeration of some other kinds of maps.
\end{abstract}

Keywords-quadrangulation; rectilinear embedding; lagrangian inversion; enumerating function

\section{INTRODUCTION}

Maps considered here are graphs embedded in a surface without edge-crossing and each face homeomorphic to an open disc, if the surface is the plane or the sphere, the map is called a planar map. A map is rooted if an edge is distinguished with an end (called root-vertex) and a side of the edge (called root). The selected edge is called root-edge. The face to which the root belongs is called root-face. A map is said to be 2-boundary if the root-face is a 2-gon. Without loss of generality, the root-face is chosen as the infinite face. All other faces are called inner faces.

A rooted planar near-quadrangulation is a rooted planar map in which each inner face is a quadrangle. If the root-face of a rooted planar near-quadrangulation is also a quadrangle, then the map is called a quadrangulation. An outerplanar nearquadrangulation can be treated as quadrangulations on the disc such that all the vertices are on the boundary of the disc. For only one quadrangulation, the quadrangle itself is outerplanar.

Quadrangulations (or their duals: rooted 4-regular planar maps) have been investigated by many scholars. Such as Tutte [1-2], Brown[3], Mullin and Schellenberg[4], Li and Liu[5]. Quadrangulations and 4-regular maps ( or quartic maps as some scholars called them) are very important, the usage can be seen for rectilinear embedding in VLSI, for the Gaussian crossing problem in graph theory, for the knot problem in topology, and for the enumeration of some other kinds of maps.

In this paper, the enumeration of rooted planar nearquadrangulations is discussed mainly with the size, the valency of the root-face and the number of non-rooted vertices as parameters. Based on this, two explicit expression of rooted planar near-quadrangulations with the size and with the root face valency are derived respectively. For one special case, rooted 2 edge-connected near-quadrangulations are considered. Moreover, we calculate rooted planar quadrangulations via rooted 2 boundary planar near-quadrangulations.

A map denoted by $M=\left(X_{\alpha, \beta}, P\right), X_{\alpha, \beta}(X)=\sum_{x \in X} K x, X$ is a finite set and $P$ is a basic permutation on $X$. For convenience, the notations and terminologies not mentioned here can be seen in Liu [6-8]. Let $Q_{1}$ be the set of all rooted planar nearquadrangulations without 2-boundary ones; $Q_{2 e}$ be the subset of $Q_{1}$ and for any $M \in Q_{2 e}, M$ is 2 edge-connected; $Q_{\mathrm{nq}}$ be the set of all general rooted planar near-quadrangulations including 2-boundary ones. $Q_{\mathrm{rvn}}$ be the set of all general rooted planar near-quadrangulations with the rooted vertex is nonseparable. For a map $M$, the root, the root-vertex, the rootedge and the root-face are denoted by $r(M), v_{r}(M)$ and $e_{r}(M)$, $f_{r}(M)$ respectively. Since the valency of all faces of planar near quadrangulations is four probably except the root-face, the root-face valency of any planar near quadrangulation has to be even. We define generating functions for $Q_{i}, i=1,2 e$, nq, rvn.

$$
f_{i}(x, y)=\sum_{M \in Q} x^{2 m(M)} y^{n(M)} z^{t(M)}
$$

where $2 m(M), n(M)$ and $t(M)$ are the valency of the rootface, the size and the number of non-rooted vertices. Further, we write that 


$$
G_{i}(x)=f_{i}(x, 1), F_{i}(y)=f_{i}(1, y), h_{i}(x, z)=f_{i}(x, 1, z)
$$

For $i=1,2 e$, nq, rvn as some special enunfunctions.

In this paper, we obtain main results as follows:

Theorem I.A. The enumerating function $f_{1}(x, y)$ satisfies the following equation

$$
x^{2} y f_{1}^{4}-\left(1-x^{-2} y\right) f_{1}^{3}+\left(1-3 x^{-2} y\right) f_{1}^{2}+3 x^{-2} y f_{1}-x^{2} y=0
$$

Furthermore,

$$
\begin{aligned}
& G_{1}(x)=1+\sum_{m \geq 1} A_{m} x^{2 m}, A_{m}=\sum_{0 \leq i \leq m-1} \frac{(2 m) !(3 m-2 i-3) !}{i !(2 m-i) !(m-i-1) !(2 m-i-1) !} \\
& F_{1}(y)=1+\sum_{n \geq 1} A_{n} y^{n}, A_{n}=\sum_{k \geq 1}^{\frac{n+2}{3}} \frac{(n-1) !(2 n-4 k+4) !}{(k-1) !(n-k+1) !(n-k+2) !(n-3 k+2) !} ;
\end{aligned}
$$

Theorem I.B. The enumerating function $f_{2 e}(x, y)$ satisfies the following equation

$$
\left(x^{2}-y\right) f_{2 e}{ }^{3}-\left(x^{2}+x^{4} y-3 y\right) f_{2 e}{ }^{2}-3 y f_{2 e}+y=0
$$

Moreover, for $i+j \leq m, 0 \leq i \leq m+20 \leq j \leq m-2$

$$
\begin{aligned}
& F_{2 e}(y)=1+\sum_{n \geq 1} B_{n} y^{n}, G_{2 e}(x)=1+\sum_{m \geq 1} B_{m} x^{2 m} ; \\
& B_{n}=\sum_{k \geq 1}^{\left.\frac{n-1}{3}\right\rfloor} \frac{(n-1) !(n-k-2) !}{k !(n-k) !(2 k-1) !(n-3 k-1) !} ; \\
& B_{m}=\sum_{i, j} \frac{3^{m-i-j} 2^{i}(m+2) !(m-2) !(2 m-i-j) !}{i ! j !(2 m-2 i+4) ! !(2 m-2 j-4) ! !(m-i-j) !}
\end{aligned}
$$

Theorem I.C. The enumerating function $h_{\mathrm{nq}}(x, z)$ satisfies the following equation

$$
x^{4} z h_{\mathrm{nq}}^{2}+\left(1-x^{2}\right) h_{\mathrm{nq}}+x^{2}-x^{2} H_{\mathrm{nq}}-1=0
$$

where $H_{\mathrm{nq}}$ is the coefficient of $x^{2}$ in $h_{\mathrm{nq}}(x, z)$. And the explicit solution of (3) is

$$
h_{\mathrm{nq}}(x, z)=\sum_{n \geq m \geq 1} C_{m, n} x^{2 m} z^{n} \quad C_{m, n}=\frac{3^{n-m}(2 m) !}{m !(m-1) !} \frac{(2 n-m-1) !}{(n-m) !(n+1) !}
$$

\section{ESTABLISHMENT OF THE EQUATIONS}

For two maps $M_{1}$ and $M_{2}$ with their respective roots $r_{1}=r\left(M_{1}\right)$ and $r_{2}=r\left(M_{2}\right)$. The map $M=M_{1} \cup M_{2}$, provided $M_{1} \cap M_{2}=\{v\}$ with $v=v_{r_{1}}=v_{r_{2}}$ is defined to have its root, root-vertex and root-edge are as the same as those of $M_{1}$, but the root-face is the composition of $f_{r_{1}}\left(M_{1}\right)$ and $f_{r_{2}}\left(M_{2}\right)$, where $f_{r_{i}}\left(M_{i}\right)$ is the root-face of $M_{i}(i=1,2)$. The operation for getting $M$ from $M_{1}$ and $M_{2}$ is called $1 v$-addition and is denoted b $M=M_{1}+M_{2}$.

Further, for two sets of maps $M^{(1)}$ and $M^{(2)}$, the set of maps $M^{(1)} \oplus M^{(2)}=\left\{M_{1}+M_{2} \mid M_{i} \in M^{(i)}, i=1,2\right\}$ is said to be the $1 v$-production of $M^{(1)}$ and $M^{(2)}$.
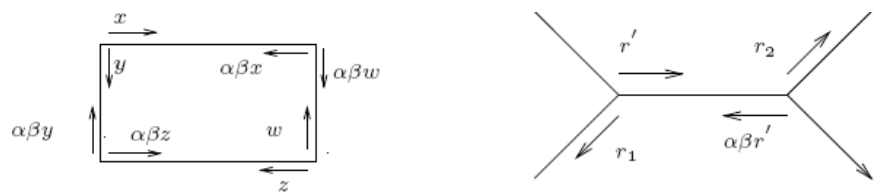

FIGURE I. QUADRANGLE AND $Q_{1}^{(\mathrm{I})}$

Here we consider $Q_{1}(x, y)$ first, which can be partitioned into three parts: Quadrangle and

1) $Q_{1}^{(0)}=\{v\}, v$ is the vertex map;

2) $Q_{1}^{(\mathrm{I})}=\left\{M \mid e_{r}(M)\right.$ is an isthmus $\}$.

The link map $L=(K r,(r)(\alpha \beta r))$ is included;

1) $Q_{1}^{(\mathrm{II})}=\left\{M \mid e_{r}(M)\right.$ belongs to a simple circuit $\}$.

$Q_{\mathrm{nq}}(x, y)$ follows the similar pattern of this partition.

Lemma II.A. Let $Q_{\langle 1\rangle}^{(\mathrm{I})}=\left\{M-a \mid \forall M \in Q_{1}^{(\mathrm{I})}\right\}$ where $a=e_{r}(M)$ is the root-edge of $M$, then $Q_{<1>}^{(\mathrm{I})}=Q_{1} \times Q_{1}$ (where $\times$ presents Descartes product between sets).

Proof: Because $\forall M \in Q_{1}^{(\mathrm{I})}$, the root-edge a of $M$ is a cut edge, $\quad M-a=M_{1}+M_{2}, M_{1}, M_{2} \in Q_{1} \quad$. That implies $M-a \in Q_{1} \times Q_{1}$. Hence $Q_{<1>}^{(\mathrm{I})} \subseteq Q_{1} \times Q_{1}$. 
Conversely, for $\forall M \in Q_{1} \times Q_{1} \quad$, we have $M=M_{1}+M_{2} \cdot M_{1}, M_{2} \in Q_{1}$. The only map $M^{\prime}=\left(X^{\prime}, P^{\prime}\right)$ can be obtained by adding an edge $\mathrm{Kr}$, which connects $v_{r}\left(M_{1}\right)$ and $v_{r}\left(M_{2}\right)$ (See Figure I). Then $M^{\prime}$ is also outerplanar quadrangulation, and $K r^{\prime}$ is a cut edge, so $\quad M^{\prime} \in Q_{1}^{(\mathrm{I})} \quad, \quad M=M^{\prime}-a \in Q_{<1>}^{(\mathrm{I})}$. This means that $Q_{1} \times Q_{1} \subseteq Q_{\langle 1\rangle}^{(\mathrm{I})}$.

From Lemma II.A, we obtained the contribution of $Q_{1}^{(\mathrm{I})}$ to $Q_{1}$ is

$$
f_{1}^{(\mathrm{I})}=x^{2} y f_{1}^{2}
$$

where $x^{2}$ presents the contribution of the root-edge to the rootface boundary of a map $M$ in $Q_{1}^{(I)}$.

\section{Lemma}

$$
\text { II.B. } \quad Q_{1}=\sum_{k \geq 0} Q_{\mathrm{rvn}}^{\oplus k}
$$

Where $Q_{\mathrm{rvn}}=\left\{M \mid \forall M \in Q_{\mathrm{rvn}} \subset Q_{1}, v_{r}(M)\right.$ is nonseparable $\}$.

Proof: $\forall M \in Q_{1}$, it is easily to see that $M$ has $k$ components $M_{i}(1 \leq i \leq k)$ and $M_{i} \in Q_{\mathrm{rvm}}$, such that $M=M_{1}+M_{2}+\stackrel{\bullet}{\bullet} M_{3} \ldots+\stackrel{\bullet}{\bullet}$, hence $M \in Q_{\mathrm{rvn}}^{\oplus k}$.

From Lemma II.B, $f_{1}=\sum_{k \geq 0} f_{\mathrm{rvn}}^{k}=\frac{1}{1-f_{\mathrm{rvn}}} \quad$ by simplification we obtained that $f_{\mathrm{rvn}}=\frac{f_{1}-1}{f_{1}}$.

For a map $M \in Q_{1}^{(\mathrm{II})}, a=k r$ is the root-edge of $M$, let $M \wedge a$ be the map obtained by deleting the edge $a$, such that the root of $M=a$ is $(P \alpha \beta P r)$, where $\left(\operatorname{Pr}, P \alpha \beta P r,(P \alpha \beta)^{2} \operatorname{Pr}, a\right)$ is the quadrangle incident to $a$.

Lemma II.C. Let $Q_{\langle 1\rangle}^{(\mathrm{II})}=\left\{M-a \mid \forall M \in Q_{1}^{(\mathrm{II})}\right\}$.

Then $Q_{\langle 1\rangle}^{(\mathrm{II})}=\left(Q_{1}-Q_{1}^{(0)}\right) \times Q_{\mathrm{rvn}}^{2}$

Proof: $\forall M^{*}=\left(X^{*}, P^{*}\right) \in\left(Q_{1}-Q_{1}^{(0)}\right) \times Q_{\mathrm{rvn}}^{2}$, for the rootvertex $v_{r^{*}}$ is a cut vertex, we may obtained a map
$M=M^{*} \hat{+} a^{*}$ by join an edge $a^{*}=\left(v_{(P \alpha \beta)^{2 m-1} r^{*}}, v_{(P \alpha \beta)^{2} r^{*}}\right)$ where the root-face of $M^{*}$ is $\left(r^{*}, P \alpha \beta r^{*}, \cdots \cdots,(P \alpha \beta)^{2 m-1} r^{*}\right)$. Since $a^{*}$ is an edge on a circuit, so $M=M^{*} \hat{+} a^{*} \in Q_{1}^{(\mathrm{II})}$ (See Figure II), it is easily checked that $M^{*}=M \sim a^{*}$.

By considering that the valency of the root-face of a map in $Q_{1}^{(I I)}$ is 2 less than that of the corresponding map in $\left(Q_{1}-Q_{1}^{(0)}\right) \times Q_{\mathrm{rvn}}^{2}$, we have $Q_{1}^{(\mathrm{II})}$ to $Q_{1}$ is $f_{1}^{(\mathrm{II})}=x^{-2} y\left(f_{1}-1\right) f_{\mathrm{rvn}}^{2}$. For $f_{\mathrm{rvn}}=\frac{f_{1}-1}{f_{1}}$, we get that

$$
f_{1}^{(\mathrm{II})}=x^{-2} y\left(f_{1}-1\right)\left(\frac{f_{1}-1}{f_{1}}\right)^{2}
$$

Since $f_{1}=f_{1}^{(0)}+f_{1}^{(\mathrm{I})}+f_{1}^{(\mathrm{II})}$, from $f_{1}^{(0)}=x^{0} y^{0}=1$ and (4), (5), we have

$$
f_{1}=1+x^{2} y f_{1}^{2}+x^{-2} y\left(f_{1}-1\right)\left(\frac{f_{1}-1}{f_{1}}\right)^{2}
$$

Multiplying by $f_{1}^{2}$ the two sides, Theorem I.A (1) follows from some rearrangement.

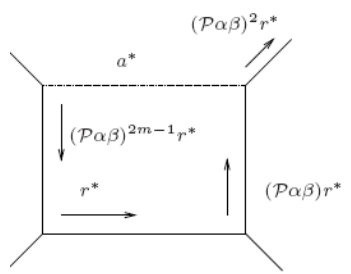

FIGURE II. $\quad Q_{1}^{(\text {II) }}$

Here we consider $Q_{\mathrm{nq}}(x, y)$ in the same way as $Q_{1}(x, y)$, which can be partitioned into three parts.

Lemma II.D. Let $Q_{(\mathrm{nq})}^{(\mathrm{I})}=\left\{M \bullet e_{r}(M) \mid M \in Q_{\mathrm{nq}}^{(\mathrm{I})}\right\}$.

Then $Q_{(\mathrm{nq})}^{(\mathrm{I})}=Q_{\mathrm{nq}} \oplus Q_{\mathrm{nq}}$

Proof: $\forall M \in Q_{\mathrm{nq}} \oplus Q_{\mathrm{nq}}$, there exists $M=M_{1}+M_{2}, M_{1}$, $M_{2} \in Q_{\mathrm{nq}}$. After splitting the root-vertex of $M$, it will result in a map $M^{\prime}$ in $Q_{\mathrm{nq}}^{(\mathrm{I})}$ such that $M=M^{\prime} \cdot e_{r}\left(M^{\prime}\right)$, this procedure is reversible. 
From Lemma II.D, we see that the contribution of $Q_{\mathrm{nq}}^{(\mathrm{I})}$ to $Q_{\mathrm{nq}}$ is $h_{\mathrm{nq}}^{(\mathrm{I})}=x^{2} z h_{\mathrm{nq}}{ }^{2}$.

Lemma II.E. Let $Q_{\langle\mathrm{nq}\rangle}^{(\mathrm{II})}=\left\{M-e_{r}(M) \mid M \in Q_{\mathrm{nq}}^{(\mathrm{II})}\right\}$, then $Q_{\langle\mathrm{nq}\rangle}^{(\mathrm{II})}=Q_{\mathrm{nq}}-v-Q_{\mathrm{nq} 2}$, where

$$
Q_{\mathrm{nq} 2}=\left\{M \mid \forall M \in Q_{\mathrm{nq} 2} \subset Q_{\mathrm{nq}} \text { the valency of } M \text { is } 2\right\} \text {. }
$$

Proof: $\forall M \in Q_{\langle\mathrm{nq}\rangle}^{(\mathrm{II})}, M=M^{*}-e_{r^{*}}\left(M^{*}\right), M^{*} \in Q_{\mathrm{nq}}^{\mathrm{II}}$. Since $e_{r^{*}}\left(M^{*}\right)$ is on a circuit, so the valency of $f_{r}(M)$ is not less than 4 . Conversely, the only map $M$ can be constructed by

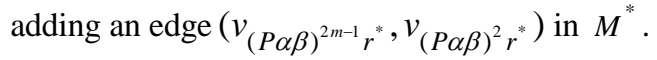

Let $H_{\mathrm{nq}}$ be the coefficient of $x^{2}$ in $h_{\mathrm{nq}}(x, z)$.

From Lemma II.E, we have

$$
h_{\mathrm{nq}}^{(\mathrm{II})}=x^{-2}\left(h_{\mathrm{nq}}-1-x^{2} H_{\mathrm{nq}}\right)
$$

Since $h=h_{\mathrm{nq}}=h_{\mathrm{nq}}^{(0)}+h_{\mathrm{nq}}^{(\mathrm{I})}+h_{\mathrm{nq}}^{(\mathrm{II})}$, from $h_{\mathrm{nq}}^{(0)}=x^{0} z^{0}=1$, $h_{\mathrm{nq}}^{(\mathrm{I})}=x^{2} z h_{\mathrm{nq}}{ }^{2}$ and (6), we get

$$
h_{\mathrm{nq}}=1+x^{2} \mathrm{zh}_{\mathrm{nq}}^{2}+x^{-2}\left(h_{\mathrm{nq}}-1-x^{2} H_{\mathrm{nq}}\right)
$$

By rearranging the terms, we soon find the equation (3) of Theorem I.C.

\section{PARAMETRIC EXPRESSIONS}

Although from (1) it is allowed to find $f_{1}$ directly by using Lagrangian inversion, because of the equation is quadruple, the result is rather complicated for usage. In order to find the enumerating explicit expressions, we have to do some transformation and find their parametric expressions firstly.

For the generating function $f=f_{1}$ satisfies then $F=f_{1}(1, y)$ satisfies

$$
F^{4}+\left(1-\frac{1}{y}\right) F^{3}+\left(\frac{1}{y}-3\right) F^{2}+3 F=1
$$

Because the left side of the equation (7) has the following decomposition, so we write

$$
F^{3}+\left(2-\frac{1}{y}\right) F^{2}-F+2=\frac{1}{1-F}=1+F+F^{2}+F^{3}+\frac{F^{4}}{1-F}
$$

which is in fact the form as

$$
\left(\frac{1-F}{F}\right)^{2}=\frac{1}{y}+\frac{F^{2}}{1-F}
$$

Lemma III.A. The generating function $F=f_{1}(1, y)$ has the parametric expressions as follows:

$$
\left\{\begin{array}{l}
F=\frac{1}{1-t} \\
y=\frac{t(1-t)}{t^{3}(1-t)+1}
\end{array}\right.
$$

Proof: From equation (8), we introduce one parameter for $F$, let $t=\frac{1-F}{F}$ then the parametric expression is derived.

By Ren [9], according to the duality, the (3) have the following parametric expressions:

$$
\left\{\begin{array}{l}
z=\theta \frac{(2-3 \theta)}{4} \\
h=\frac{x^{2}-1+\left(1+u x^{2}\right)\left(1-v x^{2} z\right)^{\frac{1}{2}}}{2 x^{4} z}
\end{array}\right.
$$

where, $\left\{\begin{array}{l}-2=-v z+2 u, 4 z+1=-2 u v z+u^{2} \\ -u^{2} v z=-4 y(1+q), \theta=u+1\end{array}\right.$.

\section{EXPLICIT FORMULAE}

In this section, we enumerate the explicit expressions of the enumerating functions $F=f_{1}(1, y)$ by employing Lagrangian inversion based on the results as described above.

For the sake of brevity, let

$$
\partial_{x}^{i}= \begin{cases}\left.\frac{1}{i !} \frac{d^{i}}{d x^{i}}\right|_{x=0} & i \geq 0 \\ \left.x^{-i}\right|_{x=\infty} & i<0\end{cases}
$$

which is called the coefficient operator [10], or the partial, here we use the notation 


$$
\partial_{x}^{i} f=\left[x^{i}\right] f
$$

In (9), we can see that $t=y \varphi(t)$ where $\varphi(t)=t^{3}+\frac{1}{1-t}$. By applying Lagrangian inversion with one parameter,

$$
\left[y^{n}\right] F=\frac{1}{n}\left[t^{n-1}\right]\left(\varphi^{n}(t) \bullet \frac{d F}{d t}\right)
$$

We get

$$
\begin{aligned}
{\left[y^{n}\right] F } & =\frac{1}{n}\left[t^{n-1}\right]\left(\frac{1}{(1-t)^{n+2}}\left(1+t^{3}(1-t)\right)^{n}\right) \\
& =\frac{1}{n}\left[t^{n-1}\right] \sum_{k \geq 0}^{n}\left(\begin{array}{l}
n \\
k
\end{array}\right) t^{3 k}(1-t)^{k-n-2} \\
& =\frac{1}{n}\left[t^{n-3 k-1}\right] \sum_{k \geq 0}^{n}\left(\begin{array}{l}
n \\
k
\end{array}\right)(1-t)^{k-n-2} \\
& \left.=\frac{1}{n} \sum_{k \geq 0}^{\frac{n-1}{3}}\right\rfloor\left(\begin{array}{l}
n \\
k
\end{array}\right)\left(\begin{array}{c}
2 n-4 k \\
n-3 k-1
\end{array}\right)
\end{aligned}
$$

So

$$
F(y)=\frac{1}{n} \sum_{k \geq 0}^{\left.\frac{n-1}{3}\right\rfloor}\left(\begin{array}{l}
n \\
k
\end{array}\right)\left(\begin{array}{c}
2 n-4 k \\
n-3 k-1
\end{array}\right) y^{n}
$$

which is equivalent to Theorem I.A. According to this theorem, we compute the coefficients $A_{n}$ of $F(y)$, which are shown in TABLE I with respect to edges within 20 edges.

By Euler Formula, for any planar near quadrangulation, if the valency of root face is $2 m$ and the number of non-root vertices is $n$, we can derived the edge number is $2 n-m$. Therefore, $h_{\mathrm{nq}}$ can be expanded as

$$
f_{\mathrm{nq}}=\sum_{n \geq m \geq 1} C_{m, n} x^{2 m} y^{2 n-m} z^{n}
$$

\section{Corollary IV.A. (Reference [6])}

Let $T$ be the enumerating function of rooted planar trees, then $T=\sum_{n \geq 1} \frac{(2 n) !}{n !(n+1) !} y^{n}$

Proof: Notice that if $m=n$ in $C_{m, n}$, then the expression of $T$ is obtained.
TABLE I. NUMBERS OF OUTERPLANAR QUADRANGULATION

\begin{tabular}{|l|l|}
\hline $\mathrm{x}$ & \multicolumn{1}{|c|}{$A_{n}$} \\
\hline 0 & 1 \\
1 & 1 \\
2 & 2 \\
3 & 5 \\
4 & 15 \\
5 & 48 \\
6 & 160 \\
7 & 552 \\
8 & 1953 \\
9 & 7044 \\
10 & 25806 \\
11 & 95765 \\
12 & 359216 \\
13 & 1359767 \\
14 & 5187754 \\
15 & 19927572 \\
16 & 77006278 \\
17 & 299153776 \\
18 & 1167638982 \\
19 & 4576743657 \\
20 & 18007689546 \\
\hline
\end{tabular}

Let $F_{\mathrm{nq}}(y)=\sum_{n \geq 0} C_{n} y^{n}$ denote the rooted general planar nearquadrangulation with edge as a parameter, from (10), we see that

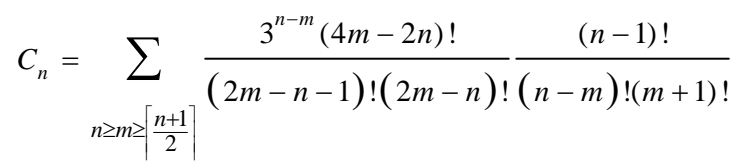

Further, let $N_{\mathrm{q}}(n)$ be the number of rooted general planar quadrangulation with $n$ edges, $N_{\text {nq2 }}(n+1)$ be the number of $Q_{\mathrm{nq} 2}$, rooted planar near quadrangulation with $n+1$ edges and the valency of the root-face is 2 .

Based on the discussion above, so the corollary follows.

Corollary IV.B. $F_{\mathrm{nq} 2}=\sum_{n \geq 1}\left(C_{n}-A_{n}\right) y^{n}$

Corollary IV.C. $N_{\mathrm{nq} 2}(n+1)=N_{\mathrm{q}}(n)$

Proof: For $\forall M \in Q_{\mathrm{nq} 2}$ can be obtained by replacing the root-edge with an additional edge on maps in $Q_{q}$, forming a multi-edge to the root-edge as the root-face boundary. On the other hand, $\forall M \in Q_{q}, M$ can be got by shrinking the multiedge on the boundary of maps in $Q_{\mathrm{nq} 2}$. 


\section{ACKNOWLEDGMENT}

This work is supported by NSFC(Natural Science Foundation of China) under Grant 60373010 and 10571013.

\section{REFERENCES}

[1] W.T.Tutte, “A census of planar maps”,Canada J.Math., vol.15, pp.249-271,1963.

[2] W.T.Tutte, "On the enumeration of planar maps", Bull.Amer.Soc., vol.74,pp 64-74,1968 .

[3] W.G.Brown, "Enumeration of quadrangular dissection of the disk”, Canad.J.Math., vol.7, pp.302-317,1965.

[4] R.C.Mullin and P.J.Schellenberg,"The number of c-nets via quadrangulations”, J.Combin.Theory.,vol.4, pp.259276,1968.

[5] Deming Li and Yanpei Liu, "The Number of 4-regular Hamiltonian Maps”, J.Northern Jiao Tong University, vol.21, pp.548-553,1997.

[6] Yanpei Liu, Enumerative Theory of Maps. Dordrecht,Boston,London:Kluwer,1999 .

[7] Yanpei Liu, Theory of Counting Combinatorial Maps [in Chinese], Beijing: Science Press,2001.

[8] Yanpei Liu, Advances in Combinatorial Maps [in Chinese], Beijing: Northern Jiaotong University Press, 2003.

[9] Han Ren, "The number of loopless 4-regular maps on the projective plane”,J. Comb. Theory, Series B, vol.84,pp.84-99,2002.

[10] I. P. Goulden and D. M. Jackson, Combinatorial Enumeation, New York: Wiley,1983. 